\title{
Molecular Modeling of Nucleic Acid Structure: Electrostatics and Solvation
}

UNITS $7.5 \& 7.8$ introduced the modeling of nucleic acid structure at the molecular level. This included a discussion of how to generate an initial model, how to evaluate the utility or reliability of a given model, and ultimately how to manipulate this model to better understand the structure, dynamics, and interactions. Subject to an appropriate representation of the energy, such as a specifically parameterized empirical force field, the techniques of minimization and Monte Carlo simulation, as well as molecular dynamics (MD) methods, were introduced as means to sample conformational space for a better understanding of the relevance of a given model. From this discussion, the major limitations with modeling, in general, were highlighted. These are the difficult issues in sampling conformational space effectively-the multiple minima or conformational sampling problems - and accurately representing the underlying energy of interaction. In order to provide a realistic model of the underlying energetics for nucleic acids in their native environments, it is crucial to include some representation of solvation (by water) and also to properly treat the electrostatic interactions. These are discussed in detail in this unit.

\section{ELECTROSTATICS AND SOLVATION}

Accurately modeling the structure and dynamics of nucleic acids with standard ab initio or empirical potentials presents special difficulties due to the highly charged phosphate backbone and the observation that nucleic acids are essentially always hydrated. Even under extremely dehydrating conditions, DNA still has very tightly associated water. To apply an accurate model, some representation of this structural and solvating water is likely necessary. Water has a structural role as both a donor and acceptor of hydrogen bonds, and can not only be specifically associated with the nucleic acid backbone but can specifically and nonspecifically associate into both the major and minor grooves. The importance of the structural water was readily observed in the first crystal structures that showed a clear spine of hydration in the minor groove (Drew and Dickerson, 1981). In addition to the structural role, water has a number of special properties. In addition to hydrodynamic properties, random thermal excitations, and viscous damping forces (which are likely to be important considerations when representing transport properties or investigating the dynamics), there are strong polarization effects. The relatively high permittivity, or dielectric constant, of water $(\sim 80)$ strongly screens the electrostatic interactions. On a microscopic level, this screening results from reorientation of permanent dipoles and electronic polarizability (or the creation of induced dipoles in the presence of an electric field). In addition to the specific structural and polarizing effects, another important interaction of the solvent derives from nonspecific entropic effects. Nonpolar molecules, those without hydrogen bonding capability or charges to interact with the water, attempt to minimize their exposure to water and tend to associate; this hydrophobic effect is a large driving force in protein folding (Dill, 1990; Spolar and Record, 1994) and appears to be a larger driving force in the association of ligands into the minor groove of DNA than electrostatic effects (Misra et al., 1994).

In addition to solvent, the high charge density from the polyionic backbone and profound salt dependence on the structure of nucleic acids are likely to necessitate some representation of ionic screening beyond simple screening by solvent. This can be included through addition of explicit counterions. However, even with the inclusion of explicit solvent and counterions in nucleic acid simulation and the use of a reasonable nucleic acid force field, if the long-range forces are not properly represented, nucleic acid

Contributed by Thomas E. Cheatham, III, Bernard R. Brooks, and Peter A. Kollman

Biophysical Analysis of Nucleic Acids

\subsection{1}

Supplement 5 
structure will be unstable during molecular dynamics simulation (Cheatham et al., 1995). This is due to the presence of high charge and large electrostatic interactions that decay only slowly with distance due to the long-range character of the Coulombic potential. Standard approximations to limit the effective range (for more tractable computation) often lead to instability (as will be discussed in greater detail later). All of these effects-i.e., improper representation of the solvent and ionic effects and improper treatment of the long-range electrostatic interactions - unduly influence the structure and dynamics of nucleic acids during simulation. However, as discussed in previous units, the level of simulation one applies represents a tradeoff in speed and accuracy, and in some cases it may be unfeasible to fully represent the effect of solvent, ions, and long-range electrostatics. Until fairly recently, computer power was not sufficient to allow fully detailed treatments with explicit water and complete representation of the long-range electrostatics. Therefore, the first MD of DNA was performed in vacuo (without any representation of screening by solvent or ions; Levitt, 1983). In order to prevent the duplex from distorting, it was necessary to remove the charge on the phosphates. Unfortunately, this approximation removes one of the essential features of polymeric nucleic acids. There are, however, some simple approximations that can be applied and that reasonably represent solvent screening; these are discussed in the next section. This will be followed by discussion of more accurate implicit solvent representations, followed finally by the more computationally demanding (but even more accurate) treatments that include explicit solvent.

\section{IN VACUO REPRESENTATIONS}

Simulations without any explicit solvent, or in vacuo simulations, are typically very rapid (with an empirical potential) since the omission of explicit solvent reduces the requisite number of atoms. Therefore, all pairwise interactions can typically be calculated, and cutoffs that limit the number of pairs included in the intermolecular interactions are not necessary. However, unless the nucleic acid environment is actually in the gas phase (where the dielectric constant, $\varepsilon$, is equal to 1), simulation tends to overemphasize the charge interactions compared to what is expected in solution. The significant phosphate repulsion will therefore tend to destabilize the structure. Although this can in part be remedied by reducing the charges on the ionic groups (as in the early MD), this is unrealistic. Alternatively, it is possible to increase the effective dielectric $(\varepsilon)$ of the system up to the permittivity of water $(\varepsilon \approx 80)$ this, however, leads to too much screening at short distances, leading to destabilization of short-range charge interactions, such as hydrogen bonds. Ideally we want a method that allows the full charges at short range $(\varepsilon=1)$ and bulk solvent screening at longer distances (i.e., $\varepsilon \approx 80$ at $20 \AA$ ). The simplest way to do this is through the application of "effective" dielectric constants or modified dielectric functions (Davis and McCammon, 1990). The most common and simplest form is the distance-dependent dielectric constant, where $\left(\varepsilon=k r_{i j}\right.$ with $k$ in the range from 1 to 4 . A better form which does not screen as drastically at short range uses a more complex sigmoidal dielectric function (Hingerty et al., 1985; Ramstein and Lavery, 1988; Daggett et al., 1991) that tapers the short-range screening more slowly. Alternatively, a dielectric function that increases exponentially with distance (consistent with Debye-Hückel theory) has been applied in the simulation of nucleic acids (von Kitzing and Diekmann, 1987; Sarai et al., 1988). A drawback of the distance-based effective dielectric functions is the uniformity of the screening regardless of the proximity to solvent or environment. This is a poor approximation for a macromolecule that tends to have a lower effective dielectric in the interior of the molecule compared to bulk. Moreover, these functions tend to cause the molecule to compact during the dynamics and suppress motion (Harvey, 1989; Steinbach and Brooks, 1994). 
Despite these issues, these treatments have been routinely applied in modeling nucleic acids. In MD simulation, these approaches lead to good representation of DNA duplexes, and when computers were less powerful, these methods were widely used (Beveridge et al., 1993). Less reasonable behavior is observed with higher-order nucleic acid structures, such as tRNA. When coupled with specifically parameterized force fields and internal coordinate treatments, sigmoidal dielectric functions provide a fast means to investigate nucleic acid structure. With simplified treatments such as this, counterion damping of the charge interactions can be accounted for effectively by reducing the charge on the phosphates or including explicit counterions. If explicit counterions are used, the standard ion parameters appropriate for solvent are not applicable; instead, larger ion radii are needed to effectively represent the first solvation shell of ion hydration (Singh et al., 1985). Recent applications of simplified internal coordinate treatments with effective dielectric constants include investigation of extreme stretching of DNA (Lebrun and Lavery, 1996; Lebrun et al., 1997) and DNA A-tract bending (Zhurkin et al., 1991). These simplified methods are powerful enough to characterize and evaluate various nucleic acid force fields in common usage (Flatters et al., 1997). In spite of these successes, it is important to note that such a simple form for the dielectric screening is unlikely to accurately represent the dielectric response of the surrounding medium, which is dependent on the position of all charges rather than a uniform scaling based on simple pairwise distances. Investigations of DNA suggest that no one form of an effective dielectric can reasonably represent all types of pair interactions (Friedman and Honig, 1992). In minimal nucleic acid models, despite the limitations, these treatments are very useful for rapid characterization of the structure. Additionally, although the DNA is not perfectly represented, this level of representation is often sufficient for use in the refinement of structure based on restraints from NMR data.

\section{IMPLICIT SOLVENT MODELS}

Although the effective dielectric functions can partially represent solvent screening, there is no representation of any reorientational polarization. In addition, the screening is unrealistically uniform and there is no representation of hydrophobic or hydrodynamic effects. To correct some of these deficiencies, various implicit solvent models may be applied. These have the benefit that the calculation of the influence of solvent is very rapid and moreover is typically represented in terms of a solvation free energy. This is possible due to the uniformity of the dielectric continuum which represents the solvent ensemble properties directly, rather than as a sampling of many distinct solvent configurations. Treating all the water as "bulk" through the use of a dielectric continuum may not be advisable in all conditions, since it is clear that some waters are structurally important and that an explicit representation is therefore necessary. However, some explicit structural water can be included along with an implicit "bulk" water representation outside the explicit system.

\section{Surface Area Approaches}

From the observation that the free energy of solvation for a saturated hydrocarbon is linearly related to the solvent-accessible surface area, one can create a set of empirical parameters to effectively represent the hydrophobic effect. For protein simulation, Eisenberg and McLachlan developed a series of effective atomic solvation parameters $\left(\sigma_{i}\right)$ based on the water/octanol transfer free energy for a variety of amino acid analogs (Eisenberg and McLachlan, 1986). Using these parameters, which implicitly include the effective polarization in the parameterization, the free energy of interaction of a given residue with water $\left(\Delta G_{\text {residue }}\right)$ is related to the change in solvent-accessible surface area of that residue (in the folded state) or $\Delta A_{i}$ :

Biophysical Analysis of Nucleic Acids

\subsection{3}

Supplement 5 


$$
\Delta G_{\text {residue }}=\sum_{\text {atoms }, i} \sigma_{i} \Delta A_{i}
$$

Scheraga and co-workers also developed this method instead using vapor/water transfer free energies (Ooi et al., 1987; Kang et al., 1988; Williams et al., 1992).

Given the derivative of the solvent-accessible surface area (or exposed van der Waals surface) necessary for calculation of the forces, this term can be included in MD simulation (Wesson and Eisenberg, 1992; Schiffer et al., 1993). In practice, depending on the speed of calculating the solvent-accessible surface area (and derivatives), adding in these terms costs anywhere from $\sim 1$ to 4 times standard MD in vacuo. The relative costs relate to the accuracy of the derivatives; exact treatments are rather expensive, but more approximate treatments (which have errors in the $1 \%$ to $5 \%$ range) can be calculated much more rapidly (LeGrand and Merz, 1993; Sridharan et al., 1995; Fraczkiewicz and Braun, 1998). Although the calculation is fairly rapid compared to explicit solvent, inclusion of these derivatives into MD simulation has not been used extensively in biomolecular simulation to date. This is because the results are very sensitive to the parameters used-a good parameter set has not emerged from the studies-and solvent screening or polarization needs to be included (either with a rudimentary distance-dependent dielectric or more complex treatments). Moreover, although it may be argued that the hydrophobic driving force for protein folding might be reasonably represented by such a term, it is not clear if this approach will prove useful in nucleic acid molecular dynamics. With nucleic acids, the stability and conformational preferences are largely due to self-association and base stacking, hydrogen bonding with the base pairs and water, and electrostatic interactions related to the phosphates, associated ions, and solvent. Since base stacking is clearly a major driving force in nucleic acid stabilization, a solvent-accessible surface area term might allow global characterization of large-scale transitions, such as a single- to double-stranded DNA transition. However, subtle conformational differences, such as between A DNA and B DNA, which differ only very slightly in solvent-accessible surface area (Alden and Kim, 1979), will probably not be accurately estimated.

\section{Adding in Effective Polarization}

Rather than fitting the solvation free energy $\left(\Delta G_{\text {sol }}\right)$ to a single property, such as the solvent-accessible surface area, one can in principle partition $\Delta G_{\text {sol }}$ into a more natural and easily developed solvent-solvent and solute-solvent polarization term $\left(\Delta G_{\mathrm{pol}}\right)$, a solute cavity or first solvation layer term related to the formation of a cavity in the solvent $\left(\Delta G_{\text {cavity }}\right)$, and a term related to immersing the uncharged solute into this cavity $\left(\Delta G_{\mathrm{vdw}}\right)$. The latter two terms, which loosely represent the hydrophobic effect, are typically represented by a solvent-accessible surface term parameterized to represent uncharged system. However, before discussing the polarization term in more detail, note that there is no way to uniquely partition the solvation free energy into these subparts, since each partition is not a state function; despite this, given appropriate parameterization, the solvation free energy can be reasonably estimated.

The simplest way to represent the effective solvent polarization is by solving the Poisson equation. The simplest case is for an isolated point charge $(q)$ within a spherical cavity of radius $r$ and dielectric $\varepsilon_{\text {int }}$ immersed in a dielectric continuum with dielectric constant $\varepsilon$. This is the Born equation (Born, 1920).

$$
\Delta G_{\mathrm{Born}}=\frac{q^{2}}{2 r}\left(\frac{1}{\varepsilon_{\mathrm{int}}}-\frac{1}{\varepsilon}\right)
$$


The value of this is a large quantity; for an isolated point charge $\left(q=1, \varepsilon_{\text {int }}=1\right)$ with a radius of $5 \AA$ immersed in a dielectric like water $(\varepsilon \approx 80)$ this represents approximately $-66 \mathrm{kcal} / \mathrm{mol}$. A similar expression for a neutral dipolar system (with total dipole moment $\mu$ assuming $\varepsilon_{\text {int }}=1$ ) was developed by Onsager (1936).

$$
\Delta G_{\text {Onsager }}=-\frac{1}{2} \mu \times\left(\frac{2(\varepsilon-1)}{2 \varepsilon+1} \frac{\mu}{r^{3}}\right)
$$

In the above form, and a number of extensions (Kirkwood, 1939; Friedman, 1975), this is also referred to as a reaction field. A more general form applicable to real molecules (i.e., not isolated point charges or dipoles) is the Generalized Born (GB) formalism. For a series of overlapping atoms with charges $q_{i}$ immersed in a dielectric continuum with dielectric constant $\varepsilon$, where $\alpha_{i j}$ are the Born radii [with $\left.\alpha_{i j}=\left(\alpha_{i} \alpha_{j}\right)^{1 / 2}\right]$ an approximate form is:

$$
\Delta G_{\mathrm{GB}}=\frac{1}{2}\left(1-\frac{1}{\varepsilon}\right) \sum_{i} \sum_{j} \frac{q_{i} q_{j}}{\left(r_{i j}^{2}+\alpha_{i j}^{2} e^{-r_{i j}^{2} /\left(2 \alpha_{i j}\right)^{2}}\right)^{1 / 2}}
$$

For superimposed charges, this gives the Born term, a Born plus Coulomb dielectric polarization for two spheres at longer distances, and, approximately, the Onsager reaction field at short range. When included with the surface area models discussed previously, this provides a very rapid and fairly accurate (depending on the parameterization) method to estimate solvation free energy for general molecules. In the context of molecular mechanics, this is the GBSA model (Still et al., 1990; Bashford and Case, 2000). This formalism has also been applied with reasonable success in the context of molecular orbital (semiempirical) theory (see Cramer and Truhlar, 1995, and references therein). This latter approach also defines a reasonable method for estimating the Born radii that is generalizable to molecular mechanics, uses the exposed van der Waals surface (instead of the solvent-accessible surface area), and calculates solvation free energies within $\sim 0.55$ $\mathrm{kcal} / \mathrm{mol}$ over a test set of 255 molecules (Hawkins et al., 1997). This is available in the program AMSOL (Hawkins et al., 1996). A drawback of standard implementations of GBSA is that often the derivatives are simplified for efficiency reasons by assuming that the effective Born radii, which depend on all the pairwise distances, are constant over a given interval during the simulation; this reduces to sampling based on an effective dielectric with forces inconsistent with the GBSA potential.

For a more complete and general treatment of the polarization by solvent, still in the context of a continuum representation (i.e., uniform bulk dielectric outside the "solute" or explicit system) or macroscopic solvent representation, the Poisson equation needs to be solved. If the effects of salt are to be included, the Poisson-Boltzmann equation can be applied. Except for some very simple cases (such as the sphere with independent ionizable groups, as discussed above, or cylinders), analytical solutions are not possible. Instead, finite difference or other numerical methods are applied. For a complete description of Poisson-Boltzmann (PB) methods, see any of a variety of reviews (Harvey, 1989; Sharp and Honig, 1990; Gilson, 1995). There are a variety of programs for solving the PB equation in common usage, such as Delphi (Gilson et al., 1987), UHBD (Madura et al., 1995), and MEAD (Bashford and Karplus, 1990), among others. Technical issues include the sensitivity to the approximating grid and radii for specifying the molecular surface and how to represent the hydrophobic effect (typically through the addition of a surface area term). Although calculation of the free energy of solvation is rapid compared to free energy perturbation in explicit solvent, solution of the PB equation is still moderately time-consuming. Therefore, although the methods have been incorporated into MD (Gilson and Honig, 1991; Sharp, 1991; Zauhar, 1991; Gilson et al., 1993), the

Biophysical Analysis of Nucleic Acids 
complexity of the calculation (and need for first derivatives) has limited calculation to short time scales, and very few reports of its use in MD of macromolecules have been reported. Issues with the representation are that the model contains no microscopic description of water (although some limited explicit water can be included) and the results are strongly dependent not only on the bulk dielectric but on the internal dielectric within the solute. For proteins and nucleic acids, controversy surrounds what, precisely, the numerical value of the effective dielectric is (or whether it is even constant; Harvey, 1989; Warshel and Aqvist, 1991). Despite this, the PB model has proven very useful in a variety of applications; a nonexhaustive list includes investigating the salt dependence of DNA ligand interaction (Misra et al., 1994), electrostatic contributions to the B-to-Z DNA transition (Misra and Honig, 1996), base stacking (Friedman and Honig, 1995), and analysis of the stability of adenine bulge DNA conformations (Zacharias and Sklenar, 1997).

Alternative methods for representing the solvent, intermediate between a continuum model and fully explicit water, are the Langevin dipole and protein-dipole Langevin dipole methods developed by Warshel and co-workers (Warshel and Levitt, 1976; Warshel and Russell, 1984; Warshel and Aqvist, 1991; Papazyan and Warshel, 1997). The idea here is that the most important effect of the solvent is the polarization rather than the detailed structural properties. Therefore, it is possible to replace the water by fixed-point polarizabilities (reorienting dipoles responding to the local electrostatic field according to the Langevin equation) on a cubic grid which is ultimately surrounded by a continuum. Since the orientation of the Langevin dipoles-based on the mean field of the local electrostatic field - depends on the local electrostatic field, these equations are typically iterated until self-consistency; however, a noniterative technique has also been applied (Lee et al., 1993). The method has been used for investigation of solvation free energies and electrostatic components in enzyme catalysis, and appears to be generally useful; however, the general simulation community has not extensively adopted its use.

In addition to the methods discussed above, other techniques for representing the interaction with the continuum are possible, such as dielectric shielding (Luo et al., 1997). An important consideration with implicit solvent models is the lack of a microscopic understanding of the role of solvent, particularly any structural or dynamic role. Since the structural role is likely to be very important in modeling endeavors, this suggests that some explicit representation of the solvent is desirable. Therefore, although the implicit solvent models can give insight into the energetics and relative stability, the lack of microscopic detail limits its utility and in fact can be misleading. For example, simulations of a DNA triplex with implicit solvent models (either with a distance-dependent dielectric constant or a much more detailed Poisson-Boltzmann treatment) predict that an antiparallel third strand with reverse Hoogsteen base pairs would be more stable than a parallel third strand with Hoogsteen base pairs in $\mathrm{d}[\mathrm{CG}-\mathrm{G}]_{7}$ triplexes, in contrast to simulation in explicit solvent and experiment (Cheng and Pettitt, 1992, 1995). Of course, the corollary to this is that results of simulations in explicit water are not necessarily better! In some cases, short simulations of DNA with distance-dependent dielectric models agree better with experiment than simulations in explicit solvent (Fritsch et al., 1993). This points out the critical role of the nucleic acid force field, solvent parameters, stability of the methods, and the myriad of details that will become apparent in the next section. A final point is that various hybrid models can also be employed that treat an interior core (solute plus some water) explicitly with an implicit representation outside this core, for example, surrounding a nucleic acid in a blob of water by a reaction field or more detailed PB

Molecular Modeling: Electrostatics and Solvation 


\section{SIMULATIONS IN EXPLICIT SOLVENT}

There are a variety of considerations that become apparent when including an explicit solvent representation, and they boil down not only to how to represent the water and how much to add, but what type of boundary conditions to apply and how to make the calculations tractable. To make the computations tractable, we want to limit the effective number of interactions both by reducing the number of waters in the simulation and by applying cutoffs to limit the pair interactions to shorter range; however, as mentioned earlier, we want to keep in mind that misrepresenting the long-range interactions can lead to artifactual behavior. When considering how much solvent to use, we want not only enough water to solvate the solute (or nucleic acid of interest), but enough (either implicit or explicit) water surrounding this to ideally represent "bulk" water away from the solute. To allow investigation of the ensemble properties in this explicit system, we want to apply as simple and efficient a model as possible while still reasonably representing the structural, dynamic, and bulk properties of water. To represent the ensemble properties now requires detailed sampling over the various possible solvent configurations. This implies that explicit solvent should only be added for simulations employing Monte Carlo or molecular dynamics methods (UNIT 7.8), because minimization with explicit solvent will trap the structure into a less representative ice structure, rather than properly represent the ensemble properties of the solvent. In these simulations, various boundary conditions can be applied and are classified as either nonperiodic boundary conditions-e.g., surrounding the explicit system by a vacuum interface or a continuum-or as periodic boundary conditions - which effectively eliminate this interface.

The most commonly used explicit water models are the rigid three-point water models such as TIP3P (Jorgensen et al., 1983) and SPC/E (Berendsen et al., 1987). These are the simplest models that can be applied that still retain the structural, energetic, and dynamic properties of water; for example, these models adequately reproduce the density, interaction energy, and first peak of the radial distribution function for water. The rigidity of the model requires the addition of constraints during molecular dynamics simulation; this is typically performed with the SHAKE procedure (Ryckaert et al., 1977). Although these are not necessarily the best water models, they are the most widely used. Drawbacks of the three-point models are an underestimation of compressibility, absence of much structure beyond the first peak of the radial distribution function, and less tetrahedrality than expected. The latter could have implications in simulation, since, although the bulk properties are acceptable, the reduced tetrahedrality could have structural implications. It should also be noticed that the two commonly used models were parameterized with different observables in mind. Although the TIP3P model accurately reproduces the interaction energies, it diffuses roughly twice what is expected. SPC/E, on the other hand, diffuses at the expected rate at the expense of slightly higher interaction energies. Neither of these models are polarizable, and therefore they are effectively prepolarized (through a larger fixed dipole moment); this is compatible with the pairwise force fields which do not include explicit polarization but implicitly represent the polarization with enhanced charges. Although slightly better water properties are obtained with the rigid four-point TIP4P (Jorgensen et al., 1983) model (which adds an extra charge on the OH bisector $0.15 \AA$ from the oxygen), this model has not seen extensive usage in biomolecular simulation. In addition to the rigid water models, there have been reports of a variety of flexible water models; however, again these models have not seen extensive usage. As a final point, it should be noted that the commonly employed water models were parameterized for use with rather small cutoffs (in the 7 to $10 \AA$ range). Yet, with the prescribed or even longer cutoffs, and the application of reliable cutoff methods such as atom-based force shifting in the 12 to $14 \AA$ range, the neglect of the long-range electrostatic interactions can lead to artifacts in the transport properties and unexpected structure in

Biophysical Analysis of Nucleic Acids 
the radial distribution function near the cutoff (Feller et al., 1996). This is particularly notable in simulations of polyionic systems. As will be discussed, much better behavior is instead observed in simulations that do not neglect the long-range electrostatic interactions.

To make the calculations tractable, in practice the solute (or nucleic acid of interest) is only surrounded by roughly 5 to $20 \AA$ of water in each direction. While this may appear to be a minimal representation, especially given the high charge typical on nucleic acids, good behavior is observed. Molecular dynamics simulations with as little as $5 \AA$ of water surrounding the DNA in each direction give similar average structures and properties in nanosecond-length simulations compared to simulations with more water (Cheatham and Kollman, 1998; Norberto de Souza and Ornstein, 1997). Moreover, although it might be expected that the nucleic acid has far-reaching influence on the neutralizing counterion distribution, Manning theory suggests that the necessary neutralizing ions are contained within $\sim 7 \AA$ of the surface of duplex DNA, with "bulk" counterion distribution outside this range (Manning, 1978; Jayaram and Beveridge, 1996). Although the observation from these simulations and arguments about the nearness of the neutralizing counterion distribution can in part justify systems with as little as $\sim 5 \AA$ of water surrounding the nucleic acid, $\sim 10 \AA$ is a more common and tolerable value, despite the additional cost, that allows near "bulk" water away from the solute. Slightly more solvent may be necessary for nonperiodic systems, due to the surface/interface effects. Although in principle even more water may be desirable to better represent bulk solvent away from the nucleic acid and to allow effectively less concentrated solutions, the computational requirements typically limit the system size to less water.

Even with a limited amount of solvent surrounding the nucleic acid model, it is necessary to further limit the number of pairwise interactions to make the calculations tractable. This is done through the application of a cutoff that is typically applied in the 9 to $15 \AA$ range. Although this is likely an appropriate range for van der Waals interactions, it is typically insufficient for electrostatics, particularly with highly charged systems. Therefore, methods need to be applied to minimize artifacts from the truncation of the long-range electrostatic interactions (as will be discussed later).

\section{Nonperiodic Boundary Conditions}

The earliest molecular dynamics simulations of nucleic acids in explicit solvent used nonperiodic boundary conditions and surrounded a small piece of a DNA duplex, d[CGCGA], by a small blob of 806 TIP3P waters and $8 \mathrm{Na}^{+}$counterions with a vacuum interface outside of the explicit water (Seibel et al., 1985). The results from these simulations were similar to earlier in vacuo simulations, but provided the added insight that comes from a detailed investigation of hydration. Although "good" behavior was observed, this 100-psec-length simulation was too short to definitively verify the methods or to sample much of the accessible conformational space. It was also too short for the instabilities due to the cutoff of the electrostatic interactions to become apparent. Despite the apparent stability, however, even in this short run the water blob had distorted to such a degree that the terminal residues on one side of the duplex were nearly exposed to the vacuum. This can be prevented in principle by restraining the solute to remain at the center of the blob with spherical boundary potentials added at the surface to prevent solvent evaporation. The major issue with this type of representation is that the small size of the solvent blob, coupled with the reorientation of the surface waters in response to the vacuum interface, leads to a large surface tension. This surface tension, compounded by the standard boundary potentials added to prevent waters from drifting away, leads to an effectively large pressure at the center of the blob which can inhibit fluctuations, particularly at the center of the blob and at the surface where the waters are strongly 
ordered due to the vacuum interface (Fox and Kollman, 1996; Steinbach and Brooks, 1994). A crude estimate of the effective pressure increase is $R \Delta P \approx 15,000 \AA$-atm, where $R$ is the radius of the solvent droplet. Because of the large surface tension and large pressures, this boundary representation is not recommended unless the surface effects are modulated, either through the use of a very large solvent blob (which is unfeasible) or by applying some means to break up the surface ordering and/or restore the effective polarization.

An effective way to do this is to add stochastic forces to waters near the surface. This is shown schematically in Figure 7.9.1. This can be done via the Langevin equation (Berkowitz and McCammon, 1982) and leads to efficient simulations of proteins where only a core part of the system is solvated (Brooks et al., 1985, 1988). For proteins, this may be an adequate treatment since most of the strong ionic interactions are limited to the surface of the protein, and the water at the surface of the protein is not likely to play as large a structural and stabilizing role, nor the electrostatics as large a destabilizing role, as with nucleic acids. Nevertheless, this method has been applied in a limited fashion in the simulation of nucleic acids, e.g., to investigate the glass transition temperature of DNA (Norberg and Nilsson, 1996) and the hydration of guanyl-5'-3'-uridine (Norberg and Nilsson, 1995) in an $20 \AA$ A sphere of water. Although applying random forces can partially eliminate the surface tension, the strong influence of the vacuum interface prevents its complete elimination. The surface ordering is fairly significant, and attempts to design restraining functions that break up this surface order have led to very complicated size, water model, and system-specific expressions that only work moderately well (Essex and Jorgensen, 1995). In addition to removing the ordered water at the surface, it is also important to include polarization by the bulk solvent outside the explicit system. This is not well reproduced by the random fluctuations of the surface water in stochastic boundary treatments.

To include polarization terms in addition to radial constraints, Warshel and co-workers developed the SCAAS model (King and Warshel, 1989) to modulate the properties of the surface waters at a fixed radius of solvent. This model allows good simulation of the static (such as the radial distribution function) and dynamic (such as diffusion) properties for the water in spheres as small as 8 to $10 \AA$. The SCAAS model apparently also allows fairly size-independent charging free energies of ions in solution (King and Warshel, 1989; Aqvist, 1990); however, the fixed radius disallows characteristic density and volume fluctuations, and reportedly the total dipole of the system is unstable during the simulation. Also, in each of these cases, the solvent boundary is effectively fixed and parameterized for a particular radius. A related method has also been reported that allows the solvent boundary to move during the simulation (thereby not inhibiting solute volume

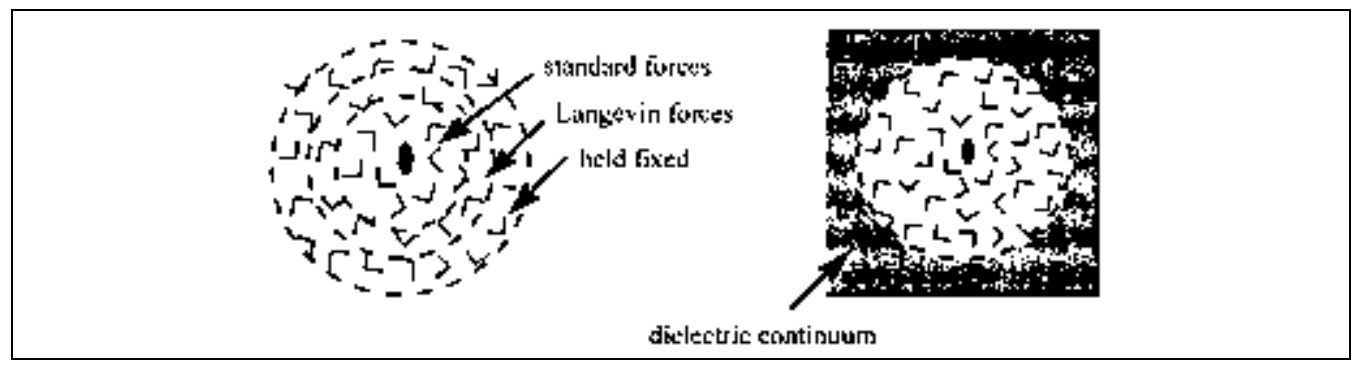

Figure 7.9.1 Representations of the system with nonperiodic boundary simulations. The picture on the left shows schematically what happens with stochastic boundary conditions, compared to a dielectric continuum, represented on the right.

Biophysical Analysis of Nucleic Acids 
changes) and models the implicit solvent outside the explicit system with a reaction field treatment (Beglov and Roux, 1994).

Given a particular implementation, these spherical "solvent boundary potentials" are parameterized to represent bulk water outside the explicit system and to minimize the surface water ordering effects. This parameterization is fairly system- and size-specific, and in the general case the model may not be appropriate unless the boundary potential is refined. A final concern is that none of the methods completely remove the effect of the interface. In spite of this, the methods have proved useful in applications ranging from ionic charging free energies and ion parameter development (Aqvist, 1990) to understanding peptide folding in solution (Mohanty et al., 1997), including the stochastic boundary applications to nucleic acid systems mentioned previously. Despite the apparent success of these methods in reproducing solvent properties and charging free energies, these methods have seen limited use in large-scale biomolecular simulation. Part of the reason is that, for biomolecular simulation, fairly large solvent spheres are necessary to completely surround the solvent. Since periodic boundary simulations can be run at a similar cost (and include shapes that are nearly spherical), periodic boundary conditions may be more appropriate. Otherwise, for small solutes and/or partially solvated macromolecules (i.e., where only a part of the system is treated in detail with explicit solvent), the solvent blobs with an appropriate boundary potential can be applied with good success.

Given the expense of the simulations and issues related to the interface between the explicit and implicit system, periodic boundary conditions may be the more appropriate choice. This is particularly true for large systems, since most of the accurate solvent blob potentials discussed above are not amenable to a standard cutoff treatment because typically all pairwise interactions are represented. This makes the calculations rather expensive. However, to counter the computational expense, various groups have recently started to apply fast multipole methods, which allow more tractable calculation of all pairwise interactions. The fast multipole method recursively groups distant atoms into multipoles based on hierarchical trees (Greengard, 1988, 1994; Greengard and Rokhlin, 1989). This tremendously increases the efficiency for large systems. For even greater efficiency (at the expense of accuracy) the cell-multipole method can also be applied (Ding et al., 1992). Ewald methods can also be adapted for simulation of finite nonperiodic systems and may provide an alternative rapid treatment for representing all pairwise interactions (Hockney and Eastwood, 1981; Pollock and Glosli, 1996).

\section{Periodic Boundary Conditions}

An artificial construction that removes the problem of the vacuum or continuum interface at the edge of the explicit system is the imposition of toroidal or periodic boundary conditions (PBC; Allen and Tildesley, 1987). This, in principle, eliminates the vacuum interface by imposing a lattice structure on the molecular system, so that, effectively, a molecule leaving one side of the periodic box enters the opposite side (see Fig. 7.9.2). With this construction, the volume and pressure are rigorously defined. Scaling of the box size (and also, typically, the relative positions of the atoms/molecules) can be used to effectively couple the pressure to define an isobaric ensemble. Similarly, scaling the velocities of the atoms can be used to control temperature, and, together, coupling the temperature and pressure allows simulation of the constant temperature and pressure ensemble (as opposed to the constant volume and energy ensemble discussed so far). Temperature and pressure scaling have typically been performed using the weak-coupling method (Berendsen et al., 1984). More recently, various simulation codes have started to incorporate more accurate coupling methods that properly simulate the isothermal/isobaric ensemble, such as Nose-Hoover thermostated chains (Nose, 1984; Hoover, 1985) or the Langevin piston method (Feller et al., 1995). 


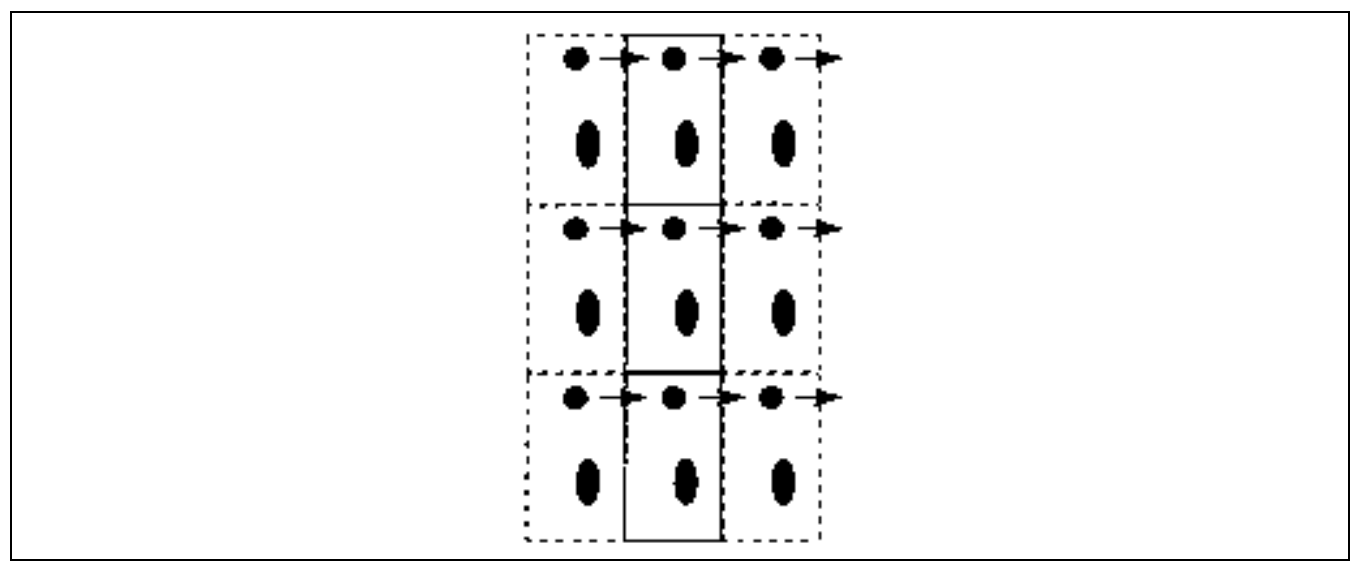

Figure 7.9.2 Periodic boundary conditions.

A potential issue with $\mathrm{PBC}$ is the imposition of true periodicity. In principle, a given atom should feel the influence of all its periodic images (see Fig. 7.9.3); for the long-range interactions, such as the electrostatics, this can be a strong influence (Valleau and Whittington, 1977). For example, we can consider a freely rotating dipole in a periodic box. Due to the influence of the periodic images, the dipole may not freely reorient. Similarly, imagine two charged particles separated by half the box length in a periodic box. Depending on the charge of the particles, they should either be attracted or repulsed. However, in a periodic lattice, due to the influence of the image particles, no net force will be felt by the particles. Additionally, due to the spatial periodicity, there may be correlation in the fluctuations or time-averaged properties; for example, a ripple or wave will not continue outward from the center unimpeded as in a nonperiodic system, but will chaotically interfere with the same ripple or wave from the image unit cells. These effects could, in principle, lead to drastic artifacts that may be more severe than the vacuum or continuum interface discussed in the previous section. In practice, with simulations including solvent with a sufficiently high permittivity (such as water) in a reasonably sized box and under equilibrium conditions, the artifacts appear minor in simulations that are truly periodic (Bader and Chandler, 1992; Smith and Pettitt, 1996; Smith et al., 1997). Also, in practice it is not necessary to treat the system as truly periodic. This is possible through the application of a cutoff that limits the pair interactions to those within the unit cell. In this way, no atom directly feels the influence of its neighbor; however, this comes at the cost of neglecting the long-range interactions.

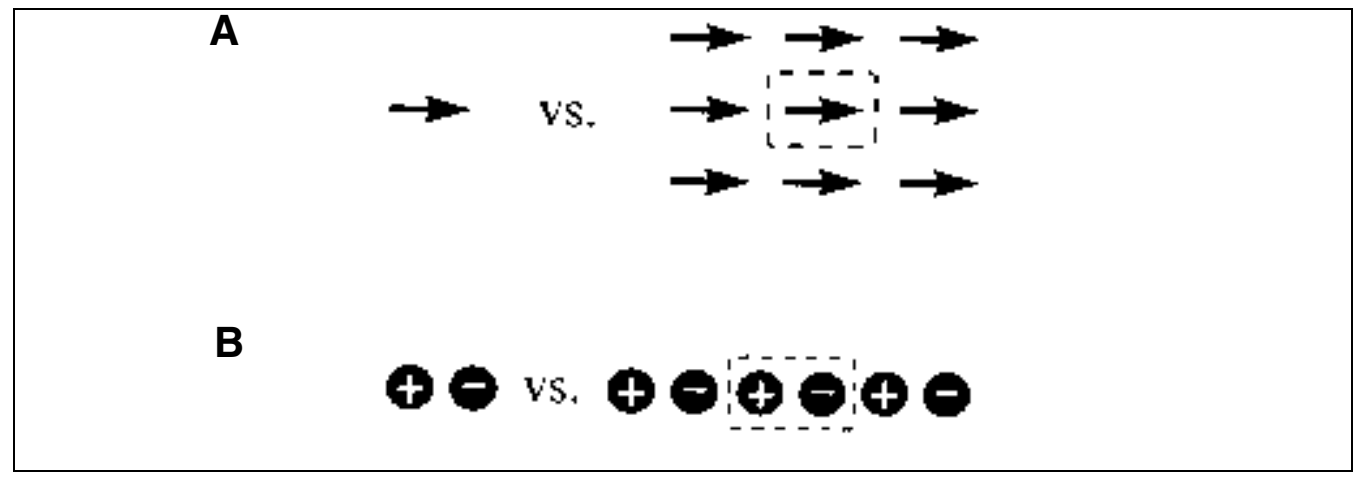

Figure 7.9.3 Potential artifacts from imposition of true periodicity. (A) Freely rotating dipole versus a dipole confined to a periodic lattice. (B) Free charges versus charges in a periodic lattice.

Biophysical Analysis of Nucleic Acids 


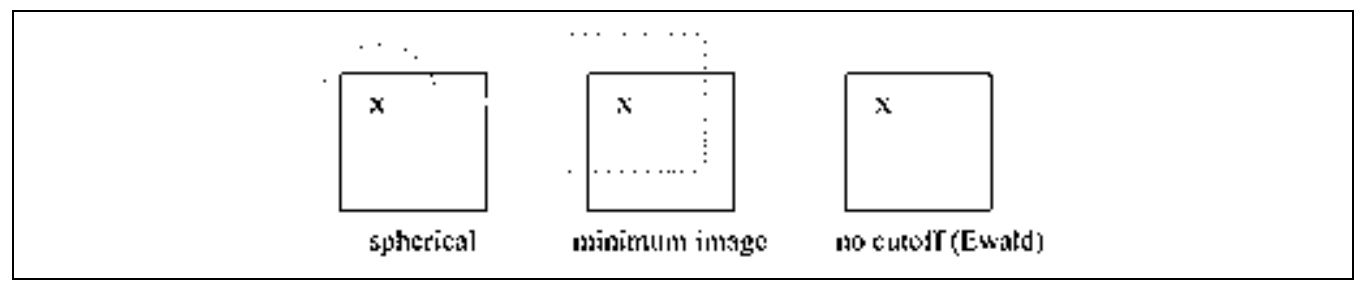

Figure 7.9.4 The various cutoff schemes.

There are two standard cutoff-truncation schemes in common usage (see Fig. 7.9.4). The standard cutoff scheme limits pair interactions about a given atom to only those atoms within a given radius. Alternatively, we can apply minimum image truncation, which includes all interactions within the unit cell centered on the given atom. However, this is not recommended because it tends to over-represent the interactions in the corners of the unit cell and leads to damped, anisotropic reorientational motion (Roberts and Schnitker, 1995). Note also that most molecular-mechanics programs, although using a radial cutoff, are limited to minimum image conditions, limiting the effective cutoff in periodic systems to half the shortest box length.

The neglect of long-range interactions leads to a number of artifacts. The largest relates to the nature of the truncation. Standard atom- or charge group-based truncation (where the latter is performed to avoid splitting up charge groups such that if one atom from the group is within the cutoff, all the atoms within that group are assumed to be within the cutoff) simply neglects all the interactions outside some finite range, typically within $\sim 8$ to $20 \AA$. At this range the electrostatic interactions are still rather large, particularly with polyionic systems such as nucleic acids. The truncation leads to force discontinuities as atoms/groups enter or leave the cutoff, and these in turn can lead to large instabilities during the dynamics. For example, even with cutoffs in the 9 to $20 \AA$ range and state-of-the-art force fields, group-based truncation leads to complete disruption of a explicitly solvated DNA duplex within $\sim 500$ psec (Cheatham et al., 1995). To avoid these problems, various techniques have been applied to smooth the discontinuity in the energy or forces, either by switching the potential (e.g., by adding a spline to bring the potential from the truncated value to zero over a short range, typically 2 to $4 \AA$ ) or shifting the entire potential to zero. However, given that the dynamics are largely dictated by the forces, these methods do not necessarily lead to better behavior. In the case of atom-based switching functions over a short range, fluctuations are completely inhibited. Much better behavior is obtained by shifting the electrostatic forces on an atom basis (and switching the van der Waals energies over a range of $\sim 2 \AA$ ); this allows stable nanosecond-length MD of nucleic acids in solution (MacKerell et al., 1995; MacKerell, 1997; MacKerell and Banavali, 2000), although water transport properties may be misrepresented (Feller et al., 1996). For a more thorough discussion of cutoffs, switching, and shifting, see the published reports (Steinbach and Brooks, 1994; Levitt et al., 1995). In addition to smoothing the interactions to the cutoff, it might be expected that better behavior will be seen with larger cutoffs. To this end, dual-cutoff or multiple-time-step methods can be applied, which treat the short-range interactions in the typical fashion but also keep track of interactions out to a larger cutoff and update these longer-range interactions less frequently (Tuckerman et al., 1992; Biesiadecki and Skeel, 1993). However, the artifactual behavior of the cutoff is not monotonically related to the size of the cutoff (i.e., longer is not necessarily better) and is not completely eliminated by smoothing the force artifacts.

Molecular Modeling: Electrostatics and Solvation
Despite the presence of significant artifacts from neglect of the long-range electrostatic interactions, cutoff approximations have been widely applied in the simulation of biomolecules such as nucleic acids. Part of the reason for this is the large size of typical 
biomolecular systems, which necessitates the use of cutoffs to limit the number of pair interactions to make the calculations more tractable. Until more recently, the time scales of the simulations $(\sim 100 \mathrm{psec})$ were not long enough to expose the deficiencies. Despite the neglect of long-range interactions, reasonable simulation of proteins was routinely observed in the earlier simulations and still continues to date (even with rather poor cutoff methods). This was not true of nucleic acid simulations, which were routinely plagued by instabilities and necessitated tricks to keep the nucleic acid stable, such as WatsonCrick hydrogen bonding restraints, reduced phosphate charges (McConnell et al., 1994), and restrained counterions (Tapia and Velazquez, 1997). Since it has more recently become possible to perform truly periodic simulations at a cost that is comparable to reasonable cutoff simulations, it is worthwhile to discuss the artifactual behavior that is seen when the long-range interactions are ignored, and compare this to the artifactual behavior that might be expected in truly periodic simulations (which fully treat the long-range electrostatic interactions).

The artifacts due to the neglect or absence of the long-range electrostatic interactions are well known and include long-range orientational correlation, such as a strong anticorrelation of dipolar fluctuations (Neumann, 1983), and decreased rotational and translational motion (van Gunsteren et al., 1978). Additionally, ion pairing between chloride ions has been observed (Dang and Pettitt, 1987), as has an attractive potential of mean force between two $\mathrm{Fe}^{2+}$ ions in solution resulting from the cutoff (despite the use of a spline smoothed potential at the cutoff; Bader and Chandler, 1992). Despite the imposition of true periodicity (which might be expected to give force artifacts due to interaction with the periodic images), Ewald methods show the expected behavior (Bader and Chandler, 1992) and no $\mathrm{Cl}^{-}$ion pairing (Hummer et al., 1993). In addition to the force artifacts, cutoff methods (with or without switched potentials) massively distort the electrostatic potential around charged groups in comparison with what is expected, in contrast to Ewald methods (Smith and Pettitt, 1991). Perhaps the most dramatic example of the problems with cutoffs, which further demonstrate that the effect of truncation is not monotonically related to the length of the cutoff, is shown in simulations of an $\alpha$-helical peptide by Schreiber and Steinhauser (1992a). In these simulations, the helix was unstable at an unreasonably short cutoff ( $6 \AA$ ), as might be expected. The helix was stable at an intermediate and commonly employed cutoff $(10 \AA)$, similar to what was seen in Ewald simulations. However, the helix was again unstable at a longer cutoff (14 $\AA$ ). What is particularly interesting in this case is the fortuitous agreement with Ewald simulations when an intermediate cutoff value was used. This is not restricted to solvated protein simulation, as fortuitous agreement has also been seen in simulation of ions in water where the orientational correlation functions and transport properties were in good agreement with results from Ewald simulation (Roberts and Schnitker, 1995). This fortuitous cancellation of errors masks the deficiencies, and has further rationalized the use of cutoff methods in protein simulation. However, for polyionic systems, this fortuitous agreement is not typically seen. For example, in salt solution the correlations between like charged ions lead to higher than expected radial populations near the cutoff, compared to a corresponding depletion of oppositely charged ions (Auffinger and Beveridge, 1995). Also, as mentioned previously, except when the forces are smoothed at the cutoff, DNA duplexes will tend to distort (Cheatham et al., 1995). However, even with smooth forces at the cutoff, such as with the application of an atom-based force shifting method, long-range order appears in the radial distribution function, and lower translational diffusion and greater viscosity of water than expected are seen; this behavior is not seen in Ewald simulations (Feller et al., 1996). The presence of all these artifacts argues for the routine use of truly periodic methods or, alternatively, the inclusion of an implicit representation of the long-range electrostatics outside the cutoff. While the former may seem drastic, the lack of artifacts in simulations to date and the relative speed have made

Biophysical Analysis of Nucleic Acids 
truly periodic methods the new "standard." In spite of this, there has been some use of reaction field methods that partially offset some of the effects of the cutoff (even within the context of periodic boundary conditions). For free energy simulations, a simple Born correction may be appropriate. More generality comes from application of a reaction field within periodic boundary simulations to represent the interactions outside the cutoff (Schreiber and Steinhauser, 1992b; van Gunsteren et al., 1978; Tironi et al., 1995, 1997) despite the potential for force/energy instabilities from molecules entering or leaving the cutoff and difficulties representing net-charged systems. These methods have seen limited usage in biomolecular simulation.

\section{Ewald and True Periodicity}

To move beyond minimum image conventions in periodic systems, it is necessary to extend the long-range interactions to a sum over the unit cells in the lattice. For truly periodic methods, this involves adding more and more unit cells into the summation until the potential converges. The electrostatic interactions are a special case, since the sum is conditionally convergent. In other words, the limiting value depends on the order of summation (for example, summing by adding more and more 2-D slabs, versus adding more and more spherical layers around the unit cell, leads to a different result). A method to evaluate the infinite sum is via the Ewald method (Ewald, 1921; Allen and Tildesley, 1987). This converts the infinite sum into a sum of a self energy plus two absolutely converging series-the screened real space or direct space interactions, and a series in reciprocal space-with an additional term (which represents the conditionally convergent part of the series) that depends on the surface and the shape of the sum at the limits. This later term, which results when the summation is represented by adding roughly spherical layers, depends on the dielectric boundary in the limit and the dipole of the unit cell (DeLeeuw et al., 1980). Under tin-foil boundary conditions, an infinite dielectric is assumed at the boundary (also called conducting boundary conditions) and this surface term vanishes. This is likely to be the appropriate boundary condition for liquids (since the liquid surface structure or polarization should disappear in the limit), whereas a dielectric boundary with dielectric constant $\varepsilon=1$ may be more appropriate for crystals. Tin-foil boundary conditions are also appropriate in net-charged systems (due to the ill-defined dipole; Bogusz et al., 1998) and are the most common dielectric boundary conditions (since this surface term is zero). In practice, application of these boundary conditions does not appear to lead to significant artifacts in the simulation of nucleic acids. However, recent simulations demonstrate that these boundary conditions may overstabilize the correlation between dipoles at long distances; this suggests using dielectric boundary conditions that more closely match the system, depending on what properties are to be represented in the simulation (Boresch and Steinhauser, 1997).

The bulk of the Ewald computation relates to the calculation of the direct and reciprocal space sums. The direct space sum represents the standard Coulombic potential screened to short range through the complementary error function, or erfc(). Since this has the same form as the standard Coulombic potential (with charges $q_{i}$ and $q_{j}$ ), it is often calculated in the same manner through the application of pairlists that include all the pair interactions within a given spherical cutoff.

$$
E_{\text {direct }}=\frac{1}{2} \sum_{i} \sum_{j} \sum_{|n| \neq 0} \frac{q_{i} q_{j} e r f c\left(\kappa\left|r_{i j}+n\right|\right)}{\left|r_{i j}+n\right|}
$$

Molecular Modeling: Electrostatics and Solvation

In the above equation, the inner sum is over the unit cells, $n=n_{a} A+n_{b} B+n_{c} C$ (for integers $n_{a}, n_{b}$, and $n_{c}$ where $A, B$, and $C$ are the unit cell lengths) and the prime (') in the sum over 
lattice vectors means omit the $i=j$ interactions when $n=0$ and the outer sums are over all atoms. The reciprocal space sum, so named since the summation is performed in reciprocal space, provides the remainder of the Ewald potential (correcting for the screening of the direct space sum).

$$
E_{\text {reciprocal }}=\frac{1}{2 \pi V} \sum_{m \neq 0} \frac{e^{-\pi^{2} m^{2} / \kappa^{2}}}{m^{2}}\left|S(m)^{2}\right| \text { where } S(m)=\sum_{i=1} q_{i} e^{2 \pi i m \cdot r_{i}}
$$

In the above expression, $V$ denotes the volume and $m$ the reciprocal vectors. The self energy is:

$$
E_{\text {self }}=-\frac{\kappa}{\sqrt{\pi}} \sum_{i} q_{i}^{2}
$$

The parameter $\kappa$ determines the width of the screening potential (typically a gaussian) that partitions the work between the reciprocal and direct space interactions. For a smaller $\kappa$ value, more of the work is in the direct space interactions; this implies that a longer cutoff is necessary to accurately represent this term. With larger $\kappa$ values, more of the work is partitioned into the reciprocal space. This requires more reciprocal vectors for the reciprocal sum (and shorter cutoffs in the direct space) for equivalent accuracy. More information on the relative speed and accuracy can be found in the published reports (Petersen, 1995; Pollock and Glosli, 1996; Toukmaji and Board, 1996; Darden et al., 1997). The additional work due to the explicit sum over the reciprocal vectors increases the computational cost of Ewald sums (optimally order $N^{3 / 2}$ where $N$ is the number of particles). Until fairly recently, due to the increased computational cost, simulation with Ewald potentials were limited to fairly small systems.

A significant advance in the past few years has been the emergence of fast Ewald methods. These significantly speed up the calculation of the Ewald sum by utilizing fast Fourier transforms (FFT) to speed the calculation of the reciprocal space interactions. Various methods have emerged, such as the particle mesh Ewald (Darden et al., 1993; Essmann et al., 1995), the particle-particle particle mesh Ewald (Luty et al., 1995; Pollock and Glosli, 1996), and the fast Fourier Poisson (York and Yang, 1994) methods, all of which are generalizations of the formulation by Hockney and Eastwood (1981). The speedup is made possible by effectively interpolating the irregularly spaced reciprocal space charges onto a regular grid, which then allows an FFT to perform the necessary convolutions. Achieving comparable accuracy to standard Ewald methods requires interpolating grids of sufficient density and reasonable interpolation of the charges; when this is done, equivalent accuracy and energy conservation are maintained. The use of the FFT changes the scaling of the Ewald method down to $N \log (N)$, which significantly increases the relative performance. In fact, since shorter cutoffs can be used for the direct space interactions $(\sim 9 \AA)$ while still completely representing the long-range electrostatic interactions, these methods are typically faster than comparable simulations with atombased force shifts at the cutoff in the 12 to $14 \AA$ range. For simulations up to $\sim 50,000$ atoms, these fast Ewald methods are the most efficient way to calculate the long-range interactions. For larger systems, periodic fast multipole methods can be applied (Schmidt and Lee, 1997), although these are slightly more complicated than standard fast multipole methods. With these methods, there is considerable controversy as to the relative accuracy and relative speed, so the 50,000-atom break-even point should not be taken as an absolute. Some of these issues are discussed in more detail in the literature (Petersen, 1995; Solvason et al., 1995; Challacombe et al., 1997). To date, almost all of the realistic simulations of biomolecular systems in the nanosecond time range that have applied a truly periodic method have involved the fast Ewald methods, and in particular the particle

Biophysical Analysis of Nucleic Acids 
mesh Ewald method. This later code has been extensively used due to its availability in common MD codes (AMBER, CHARMM), its facility for constant pressure, and the smooth nature of the errors.

As mentioned previously, application of the truly periodic methods comes at a cost: that of imposing true periodicity where all atoms in the unit cell now freely interact with their periodic images. Fortunately, for simulations in water the imposition of true periodicity does not seem to lead to significant artifacts. Dipolar rotation is not strongly inhibited; the difference between free versus hindered motion of a model dipole in water is less than $k T$ (Smith and Pettitt, 1996) and the rotation of a small zwitterionic peptide is not inhibited (Smith et al., 1997). Also, as mentioned before, no like ion pairing is seen in water (Bader and Chandler, 1992; Hummer et al., 1993). Moreover, recent simulations show that the conformational potential of mean force of a blocked trialanine in a $26 \AA$ box is similar in a truly periodic box to that calculated in the absence of true periodicity. However, care should be taken to understand the nature of potential artifacts, particularly for simulations that only include a limited amount of water. A recent study of a helical peptide suggested that when there is insufficient solvation, the peptide is artificially stabilized into an $\alpha$-helical conformation (Weber et al., 2000). Since nucleic acids are typically well hydrated and in a dielectric medium with a sufficiently high permittivity, artifacts from the true periodicity are likely to be minor. Care should be taken with the application of these truly periodic methods in solvents with a lower dielectric, such as ethanol. However, in spite of possible artifacts from true periodicity, reasonable representation of DNA in ethanol/water mixtures has been reported in MD simulation (Cheatham et al., 1997; Sprous et al., 1998). Care should also be taken with net-charged, truly periodic systems (where, in principle, the Coulombic energy should diverge!) and particularly when the charges change during the simulation, such as in free energy perturbation simulations; this is discussed in a number of publications investigating free energy of ionic hydration (Figueirido et al., 1997; Hummer et al., 1997; Bogusz et al., 1998). For a more thorough discussion of the success of nucleic acid simulation using these methods, see the authors' detailed review (Cheatham and Kollman, 2000).

\section{LITERATURE CITED}

Alden, C.J. and Kim, S.-H. 1979. Solvent-accessible surfaces of nucleic acids. J. Mol. Biol. 132:411-434.

Allen, M.P. and Tildesley, D.J. 1987. Computer Simulation of Liquids. Oxford University Press, Oxford.

Aqvist, J. 1990. Ion-water interaction potentials derived from free energy perturbation simulations. J. Phys. Chem. 94:8021-8024.

Auffinger, P. and Beveridge, B.L. 1995. A simple test for evaluating the truncation effects in simulations of systems involving charged groups. Chem. Phys. Lett. 234:413-415.

Bader, J.S. and Chandler, D. 1992. Computer simulation study of the mean forces between ferrous and ferric ions in water. J. Phys. Chem. 96:6423-6427.

Bashford, D. and Case, D.A. 2000. Generalized Born models of molecular solvation effects. Annu. Rev. Phys. Chem. 51:129-152.

Bashford, D. and Karplus, M. 1990. pKa's of ionizable groups in proteins: Atomic detail from a continuum electrostatic model. Biochem. 29:10219-10225.

Beglov, D. and Roux, B. 1994. Finite representation of in infinite bulk system: Solvent boundary potential for computer simulations. J. Chem. Phys. 100:9050-9063.

Berendsen, H.J.C., Postma, J.P.M., van Gunsteren, W.F., DiNola, A., and Haak, J.R. 1984. Molecular dynamics with coupling to an external bath. J. Comp. Phys. 81:3684-3690.

Berendsen, H.J.C., Grigera, J.R., and Straatsma, T.P. 1987. The missing term in effective pair potentials. J. Phys. Chem. 91:6269-6274.

Molecular

Modeling:

Electrostatics and Solvation

Berkowitz, M.L. and McCammon, J.A. 1982. Molecular dynamics with stochastic boundary conditions. Chem. Phys. Lett. 90:215-217. 
Beveridge, D.L., Swaminathan, S., Ravishanker, G., Withka, J.M., Srinivasan, J., Prevost, C., Louise-May, S., Langley, D.R., DiCapua, F.M., and Bolton, P.H. 1993. Molecular dynamics simulations on the hydration, structure and motions of DNA oligomers. In Water and Biological Molecules (E. Westhof, ed.) pp. 165-225. Macmillan Press, New York.

Biesiadecki, J.J. and Skeel, R.D. 1993. Dangers of multiple time step methods. J. Comp. Phys. 109:318-328.

Bogusz, S., Cheatham, T.E. III, and Brooks, B.R. 1998. Removal of pressure and free energy artifacts in charged periodic systems via net charge corrections to the Ewald potential. J. Chem. Phys. 108:7070-7084.

Boresch, S. and Steinhauser, O. 1997. Presumed versus real artifacts of the Ewald summation technique: The importance of dielectric boundary conditions. Ber. Bunsenges. Phys. Chem. 101:1019-1029.

Born, M. 1920. Volumen der Hydratationswärme der Ione. Z. Phys. Chem. 1:45-48.

Brooks, C.L., Brunger, A., and Karplus, M. 1985. Active site dynamics in protein molecules: A stochastic boundary-molecular dynamics approach. Biopolymers 24:843-865.

Brooks, C.L. III, Karplus, M., and Pettitt, B.M. 1988. Proteins. A theoretical perspective of dynamics, structure, and thermodynamics. In Advances in Chemical Physics, Vol. 71 (I. Prigogine, and S.A. Rice, eds.). John Wiley \& Sons, New York.

Challacombe, M., White, C., and Head-Gordon, M. 1997. Periodic boundary conditions and the fast multipole method. J. Chem. Phys. 107:10131-10140.

Cheatham, T.E. III and Kollman, P.A. 1998. Molecular dynamics simulation of nucleic acids in solution: How sensitive are the results to small perturbations in the force field and environment. In Structure, Motion, Interactions and Expression of Biological Macromolecules (M. Sarma and R. Sarma, eds.) pp. 99-116. Adenine Press, Schenectady, New York.

Cheatham, T.E. III and Kollman, P.A. 2000. Molecular dynamics simulation of nucleic acids. Annu. Rev. Phys. Chem. 51:435-471.

Cheatham, T.E. III, Miller, J.L., Fox, T., Darden, T.A., and Kollman, P.A. 1995. Molecular dynamics simulations on solvated biomolecular systems: The particle mesh Ewald method leads to stable trajectories of DNA, RNA and proteins. J. Am. Chem. Soc. 117:4193-4194.

Cheatham, T.E. III, Crowley, M.F., Fox, T., and Kollman, P.A. 1997. A molecular level picture of the stabilization of A-DNA in mixed ethanol-water solutions. Proc. Natl. Acad. Sci. U.S.A. 94:9626-9630.

Cheng, Y.-K. and Pettitt, B.M. 1992. Hoogsteen versus reverse-Hoogsteen base pairing in DNA triplex helices. J. Am. Chem. Soc. 114:4465-4474.

Cheng, Y.-K. and Pettitt, B.M. 1995. Solvent effects on model d(CG-G) 7 and d(TA-T) 7 DNA triplex helices. Biopolymers 35:457-473.

Cramer, C.J. and Truhlar, D.G. 1995. Continuum solvation models: Classical and quantum mechanical implementations. In Reviews in Computational Chemistry, Vol. 6 (K.D. Lipkowitz and D.B. Boyd, eds.) pp. 1-72. VCH, New York.

Daggett, V., Kollman, P.A., and Kuntz, I.D. 1991. Molecular dynamics simulations of small peptides: Dependence on dielectric model and pH. Biopolymers 31:285-304.

Dang, L.X. and Pettitt, B.M. 1987. Chloride ion pairs in water. J. Am. Chem. Soc. 109:5531-5532.

Darden, T.A., York, D.M., and Pedersen, L.G. 1993. Particle mesh Ewald: An $N \log (N)$ method for Ewald sums in large systems. J. Chem. Phys. 98:10089-10092.

Darden, T.A., Pedersen, L.G., Toukmaji, A.Y., Crowley, M.F., and Cheatham, T.E. III. 1997. Eighth SIAM conference on parallel processing for scientific computing, Minneapolis, Minn. Society for Industrial and Applied Mathematics, Philadelphia.

Davis, M.E. and McCammon, J.A. 1990. Electrostatics in biomolecular structure and dynamics. Chem. Rev. 90:509-521.

DeLeeuw, S.W., Perram, J.M., and Smith, E.R. 1980. Simulation of electrostatic systems in periodic boundary conditions. I. Lattice sums and dielectric constants. Proc. R. Soc. Lond. A373:27-56.

Dill, K.A. 1990. Dominant forces in protein folding. Biochemistry 29:7133-7155.

Ding, H.Q., Karasawa, N., and Goddard, W.A. 1992. Atomic level simulations on a million particles: The cell multipole method for Coulomb and London nonbond interactions. J. Chem. Phys. 97:4309-4315.

Drew, H.R. and Dickerson, R.E. 1981. Structure of a B-DNA dodecamer. III. Geometry of hydration. J. Mol. Biol. 151:535-556.

Eisenberg, D. and McLachlan, A.D. 1986. Solvation energy in protein folding and binding. Nature 319:199203.

Essex, J.W. and Jorgensen, W.L. 1995. An empirical boundary potential for water droplet simulations. J. Comp. Chem. 16, 951-972.

Essmann, U., Perera, L., Berkowitz, M.L., Darden, T., Lee, H., and Pedersen, L.G. 1995. A smooth particle mesh Ewald method. J. Chem. Phys. 103:8577-8593.

Biophysical Analysis of Nucleic Acids

7.9.17

Supplement 5 
Ewald, P. 1921. Investigations of crystals by means of Roentgen rays. Ann. Phys. (Leipzig) 64:253-264.

Feller, S.E., Zhang, Y., Pastor, W., and Brooks, B.R. 1995. Constant pressure molecular dynamics simulation: The Langevin piston method. J. Chem. Phys. 103:4613-4621.

Feller, S.E., Pastor, R.W., Rojnuckarin, A., Bogusz, S., and Brooks, B.R. 1996. Effect of electrostatic force truncation on interfacial and transport properties of water. J. Phys. Chem. 100:17011-17020.

Figueirido, F., Del Buono, G.S., and Levy, R.M. 1997. On finite-size corrections to the free energy of ionic hydration. J. Phys. Chem. 101:5622-5623.

Flatters, D., Zakrzewska, K., and Lavery, R. 1997. Internal coordinate modeling of DNA: Force field comparisons. J. Comp. Chem. 18:1043-1055.

Fox, T. and Kollman, P.A. 1996. The application of different solvation and electrostatic models in molecular dynamics simulations of ubiquitin: How well is the X-ray structure "maintained." Proteins 25:315-334.

Fraczkiewicz, R. and Braun, W. 1998. Exact and efficient analytical calculation of the accessible surface areas and their gradients for macromolecules. J. Comp. Chem. 19:319-333.

Friedman, H. 1975. Image approximation to the reaction field. Mol. Phys. 29:1533-1543.

Friedman, R.A. and Honig, B. 1992. The electrostatic contribution to DNA base-stacking interactions. Biopolymers 32:145-159.

Friedman, R.A. and Honig, B. 1995. A free energy analysis of nucleic acid base stacking in aqueous solution. Biophys. J. 69:1528-1535.

Fritsch, V., Ravishanker, G., Beveridge, D.L., and Westhof, E. 1993. Molecular dynamics simulations of poly(dA)-poly(dT): Comparisons between implicit and explicit solvent representations. Biopolymers $33: 1537-1552$.

Gilson, M.K. 1995. Theory of electrostatic interactions in macromolecules. Curr. Opin. Struct. Biol. 5:216-223.

Gilson, M.K. and Honig, B. 1991. The inclusion of electrostatic hydration energies in molecular mechanics calculations. J. Comp. Aided Mol. Des. 5:5-20.

Gilson, M.K., Sharp, K.A., and Honig, B.H. 1987. Calculating the electrostatic potential of molecules in solution: Method and error assessment. J. Comp. Chem. 9:327-335.

Gilson, M.K., Davis, M.E., Luty, B.A., and McCammon, J.A. 1993. Computation of electrostatic forces on solvated molecules using the Poisson-Boltzmann equation. J. Phys. Chem. 97:3591-3600.

Greengard, L. 1988. The Rapid Evaluation of Potential Fields in Particle Systems. MIT Press, Cambridge, Mass.

Greengard, L. 1994. Fast algorithms for classical physics. Science 265:909-914.

Greengard, L. and Rokhlin, V. 1989. On the evaluation of electrostatic interactions in molecular modeling. Chem. Scrip. 29A:139-144.

Harvey, S.C. 1989. Treatment of electrostatic effects in macromolecular modeling. Proteins 5:78-92.

Hawkins, G.D., Lynch, G.C., Giesen, D.J., Rossi, I., Storer, J.W., Liotard, D.A., Cramer, C.J., and Thular, D.G. 1996. AMSOL (QCPE Bull. 16, 11), Minnesota, Minn.

Hawkins, G.D., Cramer, C.J., and Truhlar, D.G. 1997. Parameterized model for aqueous free energies of solvation using geometry-dependent atomic surface tensions with implicit electrostatics. J. Phys. Chem. B. 101:7147-7157.

Hingerty, B.E., Ritchie, R.H., Ferrell, T.L., and Turner, J.E. 1985. Dielectric effects in biopolymers: The theory of ionic saturation revisited. Biopolymers 24:427-439.

Hockney, R.W. and Eastwood, J.W. 1981. Computer simulation using particles. McGraw-Hill, New York.

Hoover, W.G. 1985. Canonical dynamics: Equilibrium phase distributions. Phys. Rev. A. 31:1695-1697.

Hummer, G., Soumpasis, D.M., and Neumann, M. 1993. Computer simulations do not support Cl-Cl pairing in aqueous $\mathrm{NaCl}$ solution. Mol. Phys. 81:1155-1163.

Hummer, G., Pratt, L.R., and Garcia, A. 1997. Ion sizes and finite-size corrections for ionic-solvation free energies. J. Chem. Phys. 107:9275-9277.

Jayaram, B. and Beveridge, D.L. 1996. Modeling DNA in aqueous solutions: Theoretical and computer simulation studies on the ion atmosphere of DNA. Annu. Rev. Biophys. Biomol. Struct. 25:367-394.

Jorgensen, W.L., Chandrasekhar, J., Madura, J.D., Impey, R.W., and Klein, M.L. 1983. Comparison of simple potential functions for simulating liquid water. J. Chem. Phys. 79:926-935.

Kang, Y.K., Nemethy, G., and Scheraga, H.A. 1988. Free energies of hydration of solute molecules. 4. Revised treatment of the hydration shell model. J. Chem. Phys. 79:926-935.

King, G. and Warshel, A. 1989. A surface constrained all-atom solvent model for effective simulations of polar solutions. J. Chem. Phys. 91:3647-3661.

Modeling:

Statics and Solvation

\subsubsection{8}


Kirkwood, J.G. 1939. The dielectric polarization of liquids. J. Chem. Phys. 7:911-919.

Lebrun, A. and Lavery, R. 1996. Modelling extreme stretching of DNA. Nucl. Acids Res. 24:2260-2267.

Lebrun, A., Shakked, Z., and Lavery, R. 1997. Local DNA stretching mimics the distortion caused by TATA box-binding protein. Proc. Natl. Acad. Sci. U.S.A. 94:2993-2998.

Lee, F.S., Chu, Z.T., and Warshel, A. 1993. Microscopic and semimicroscopic calculations of electrostatic energies in proteins by the Polaris and Enzymix programs. J. Comp. Chem. 14:161-185.

LeGrand, S.M. and Merz, K.M. 1993. Rapid approximation to molecular surface area via the use of boolean logic and look-up tables. J. Comp. Chem. 14:349-352.

Levitt, M. 1983. Computer simulation of DNA double-helix dynamics. Cold Spring Harbor. Symp. Quant. Biol. 47:251-262.

Levitt, M., Hirshberg, M., Sharon, R., and Daggett, V. 1995. Potential energy function and parameters for simulations of the molecular dynamics of proteins and nucleic acids in solution. Comp. Phys. Comm. 91:215-231.

Luo, R., Moult, J., and Gilson, M.K. 1997. Dielectric screening treatment of electrostatic solvation. J. Phys. Chem. B. 101:11226-11236.

Luty, B.A., Tironi, I.G., and van Gunsteren, W.F. 1995. Lattice-sum methods for calculating electrostatic interactions in molecular simulations. J. Chem. Phys. 103:3014-3021.

MacKerell, A.D., Jr. 1997. Influence of magnesium ions on duplex DNA structural, dynamic, and solvation properties. J. Phys. Chem. B101:646-650.

MacKerell, A.D. Jr. and Banavali, N. 2000. All-atom empirical force field for nucleic acids. 2. Application to molecular dynamics simulations of DNA and RNA in solution. J. Comp. Chem. 21:105-120.

MacKerell, A.D. Jr., Wiorkiewicz-Kuczera, J., and Karplus, M. 1995. An all-atom empirical energy function for the simulation of nucleic acids. J. Am. Chem. Soc. 117:11946-11975.

Madura, J.D., Briggs, J.M., Wade, R.C., Davis, M.E., and McCammon, J.A. 1995. Electrostatics and diffusion of molecules in solution-Simulations with the University of Houston brownian dynamics program. Comp. Phys. Comm. 91:57-95.

Manning, G.S. 1978. The molecular theory of polyelectrolyte solutions with applications to the electrostatic properties of polynucleotides. Quart. Rev. Biophys. 2:159-246.

McConnell, K.J., Nirmala, R., Young, M.A., Ravishanker, G., and Beveridge, D.L. 1994. A nanosecond molecular dynamics trajectory for a B DNA double helix - Evidence for substates. J. Am. Chem. Soc. 116:4461-4462.

Misra, V.K. and Honig, B. 1996. The electrostatic contribution to the B to Z transition of DNA. Biochem. 35:1115-1124.

Misra, V.K., Sharp, K.A., Friedman, R.A., and Honig, B. 1994. Salt effects on ligand-DNA binding. Minor groove binding antibiotics. J. Mol. Biol. 238:245-263.

Mohanty, D., Elber, R., Thirumalai, D., Beglov, D., and Roux, B. 1997. Kinetics of protein folding: Computer simulations of SYPFDV and peptide variants in water. J. Mol. Biol. 272:423-442.

Neumann, M. 1983. Dipole moment fluctuation formulas in computer simulations of polar systems. Mol. Phys. 50:841-858.

Norberg, J. and Nilsson, L. 1995. NMR relaxation times, dynamics, and hydration of a nucleic acid fragment from molecular dynamics simulations. J. Phys. Chem. 99:14876-14884.

Norberg, J. and Nilsson, L. 1996. Glass transition in DNA from molecular dynamics simulations. Proc. Natl. Acad. Sci. U.S.A. 93:10173-10176.

Norberto de Souza, O. and Ornstein, R.L. 1997. Effect of periodic box size on aqueous molecular dynamics simulation of a DNA dodecamer with particle-mesh Ewald method. Biophys. J. 72:2395-2397.

Nose, S. 1984. A molecular dynamics method for simulations in the canonical ensemble. Mol. Phys. 52:255-268.

Onsager, L. 1936. Electric moments of molecules in liquids. J. Am. Chem. Soc. 58:1486-1493.

Ooi, I., Oobatake, M., Nemethy, G., and Scheraga, H.A. 1987. Accessible surface areas as a measure of the thermodynamic parameters of hydration of peptides. Proc. Natl. Acad. Sci. U.S.A. 84:3086-3090.

Papazyan, A. and Warshel, A. 1997. Continuum and dipole-lattice models of solvation. J. Phys. Chem. B 101:11254-11264.

Petersen, H.G. 1995. Accuracy and efficiency of the particle mesh Ewald method. J. Chem. Phys. 103:36683679.

Pollock, E.L. and Glosli, J. 1996. Comments on P3M, PMM, and the Ewald method for large periodic Coulombic systems. Comp. Phys. Comm. 95:93-110.

Biophysical Analysis of Nucleic Acids

7.9.19

Supplement 5 
Ramstein, J. and Lavery, R. 1988. Energetic coupling between DNA bending and base pair opening. Proc. Natl. Acad. Sci. U.S.A. 85:7231-7235.

Roberts, J.E. and Schnitker, J. 1995. Boundary conditions in simulations of aqueous ionic solutions: A systematic study. J. Phys. Chem. 99:1322-1331.

Ryckaert, J.P., Ciccotti, G., and Berendsen, H.J.C. 1977. Numerical integration of the cartesian equations of motion of a system with constraints: Molecular dynamics of $n$-alkanes. J. Comp. Phys. 23:327-341.

Sarai, A., Mazur, J., Nussinov, R., and Jernigan, R.L. 1988. Origin of DNA helical structure and its sequence dependence. Biochemistry 27:8498-8502.

Schiffer, C.A., Caldwell, J.W., Kollman, P.A., and Stroud, R.M. 1993. Protein structure prediction with a combined solvation free energy-molecular mechanics force field. Mol. Sim. 10:121-149.

Schmidt, K.E. and Lee, M.A. 1997. Multipole Ewald sums for the fast multipole method. J. Stat. Phys. $89: 411-424$

Schreiber, H. and Steinhauser, O. 1992a. Cutoff size does strongly influence molecular dynamics results on solvated polypeptides. Biochemistry 31:5856-5860.

Schreiber, H. and Steinhauser, O. 1992b. Taming cutoff induced artifacts in molecular dynamics studies of polypeptides. The reaction field method. J. Mol. Biol. 228:909-923.

Seibel, G.L., Singh, U.C., and Kollman, P.A. 1985. A molecular dynamics simulation of double-helical B-DNA including counterions and water. Proc. Nat. Acad. Sci. U.S.A. 82:6537-6540.

Sharp, K.A. 1991. Incorporating solvent and ion screening into molecular dynamics using the finite-difference Poisson-Boltzmann approach. J. Comp. Chem. 12:454-468.

Sharp, K.A. and Honig, B. 1990. Electrostatic interactions in macromolecules: Theory and applications. Annu. Rev. Biophys. Biophys. Chem. 19:301-332.

Singh, U.C., Weiner, S.C., and Kollman, P.A. 1985. Molecular dynamics simulations of d(C-G-C-G-A)-d(TC-G-C-G) with and without "hydrated" counterions. Proc. Natl. Acad. Sci. U.S.A. 82:755-759.

Smith, P.E. and Pettitt, B.M. 1991. Peptides in ionic solution-A comparison of the Ewald and switching function techniques. J. Chem. Phys. 95:8430-8441.

Smith, P.E. and Pettitt, B.M. 1996. Ewald artifacts in liquid state molecular dynamics simulations. J. Chem. Phys. 105:4289-4293.

Smith, P.E., Blatt, H.D., and Pettitt, B.M. 1997. On the presence of rotational Ewald artifacts in the equilibrium and dynamical properties of a zwitterionic tetrapeptide in aqueous solution. J. Phys. Chem. 101B:38863890.

Solvason, D., Kolafa, J., Petersen, H.G., and Perram, J.W. 1995. A rigorous comparison of the Ewald method and the fast multipole method in two dimensions. Comp. Phys. Comm. 87:307-318.

Spolar, R.S. and Record, M.T. 1994. Coupling of local folding to site-specific binding of proteins to DNA. Science 263:777-784.

Sprous, D., Young, M.A., and Beveridge, D.L. 1998. Molecular dynamics studies of the conformational preferences of a DNA double helix in water and an ethanol/water mixture: Theoretical considerations of the A-B transition. J. Phys. Chem. B. 102:4658-4667.

Sridharan, S., Nicholls, A., and Sharp, K.A. 1995. A rapid method for calculating derivatives of solvent accessible surface areas of molecules. J. Comp. Chem. 16:1038-1044.

Steinbach, P.J. and Brooks, B.R. 1994. New spherical-cutoff methods for long-range forces in macromolecular simulation. J. Comp. Chem. 15:667-683.

Still, W.C., Tempczyk, A., Hawley, R.C., and Hendrickson, T. 1990. Semi analytical treatment of solvation for molecular mechanics and dynamics. J. Am. Chem. Soc. 112:6127-6128.

Tapia, O. and Velazquez, I. 1997. Molecular dynamics simulations of DNA with protein's consistent GROMOS force field and the role of counterions' symmetry. J. Am. Chem. Soc. 119:5934-5938.

Tironi, I.G., Sperb, R., Smith, P.E., and van Gunsteren, W.F. 1995. A generalized reaction field method for molecular dynamics simulations. J. Chem. Phys. 102:5451-5459.

Tironi, I.G., Luty, B.A., and van Gunsteren, W.F. 1997. Space-time correlated reaction field: A stochastic dynamical approach to the dielectric continuum. J. Chem. Phys. 106:6068-6075.

Toukmaji, A.Y. and Board, J.A.J. 1996. Ewald summation techniques in perspective: A survey. Comp. Phys. Comm. 95:73-92.

Tuckerman, M., Berne, B.J., and Martyna, G.J. 1992. Reversible multiple time scale molecular dynamics. J. Chem. Phys. 97:1990-2001.

Molecular

Modeling:

Electrostatics and Solvation

\subsubsection{0} In Statistical Mechanics A: A Modern Theoretical Chemistry, Vol. 5-6 (B.J. Berne, ed.), pp. 137-167. Plenum Press, New York. 
van Gunsteren, W.F., Berendsen, H.J.C., and Rullman, J.A.C. 1978. Inclusion of a reaction field in molecular dynamics: Application to liquid water. Faraday Disc. 66:58-70.

von Kitzing, E. and Diekmann, S. 1987. Molecular mechanics calculations of $\mathrm{dA}_{12}-\mathrm{dT}_{12}$ and the curved molecule d(GCTCGAAAAA)4-d(TTTTTCGAGC)4. Eur. Biophys. J. 15:13-26.

Warshel, A. and Aqvist, J. 1991. Electrostatic energy and macromolecular function. Annu. Rev. Biophys. Biophys. Chem. 20:267-298.

Warshel, A. and Levitt, M. 1976. Theoretical studies of enzyme reactions: Dielectric, electrostatic and steric stabilization of the carbonium ion in the reaction of lysozyme. J. Mol. Biol. 103:227-249.

Warshel, A. and Russell, S.T. 1984. Calculation of electrostatic interactions in biological systems and in solution. Quart. Rev. Biophys. 17:283-422.

Weber, W., Hunenberger, P.H., and McCammon, J.A. 2000. Molecular dynamics simulations of a polyalanine octapeptide under Ewald boundary conditions: Influence of artificial periodicity on peptide conformation. J. Phys. Chem. B. 104:3668-3675.

Wesson, L. and Eisenberg, D. 1992. Atomic solvation parameters applied to molecular dynamics of proteins in solution. Prot. Sci. 1:227-235.

Williams, R.L., Vila, J., Perrot, G., and Scheraga, H.A. 1992. Empirical solvation models in the context of conformational energy searches: Application to bovine pancreatic trypsin inhibitor. Proteins 14:110-119.

York, D. and Yang, W. 1994. The fast Fourier Poisson method for calculating Ewald sums. J. Chem. Phys. 101:3298-3300.

Zacharias, M. and Sklenar, H. 1997. Analysis of the stability of looped-out and stacked-in conformations of an adenine bulge in DNA using a continuum model for solvent and ions. Biophys. J. 73:2990-3003.

Zauhar, R.J. 1991. The incorporation of hydration forces determined by continuum electrostatics into molecular mechanics simulations. J. Comp. Chem. 12:575-583.

Zhurkin, V.B., Ulyanov, N.B., Gorin, A.A., and Jernigan, R.L. 1991. Static and statistical bending of DNA evaluated by Monte Carlo calculations. Proc. Natl. Acad. Sci. U.S.A. 88:7046-7050.

Contributed by Thomas E. Cheatham, III

University of Utah

Salt Lake City, Utah

Bernard R. Brooks

National Heart, Lung, and Blood Institute

Bethesda, Maryland

Peter A. Kollman

University of California

San Francisco, California

Biophysical

Analysis of

Nucleic Acids

7.9.21

Current Protocols in Nucleic Acid Chemistry

Supplement 5 